\title{
Marie-Dominique Garnier et Joana Masó (dir.), Cixous sous X. D'un coup le nom
} Saint-Denis, Presses universitaires de Vincennes, coll. «L'Imaginaire du Texte », 2010, 184 p.

\author{
Lucie Ledoux \\ Université du Québec à Montréal
}

" Le nom qu'appelle-t-on ainsi? Qu'entend-on sous le nom de nom ? Et qu'arrive-t-il quand on donne un nom ? Que donne-ton alors ? », écrivait Derrida dans Saufle nom (1993). Qu'a-t-on donné à Cixous quand on lui a transmis ce nom ? Qu'est-ce qui surgit du centre du nom, de sous le nom, de sous le X? Cixous sous les rayons X... Le X est-il le cœur du nom, le nom qui dit toujours non, la signature de l'illettré? Est-ce une formule mathématique, un matricule? Chiasme ou croisement ? Lettre 
qui marque sa place? Le X représente la relation directe ou indirecte qu'entretient cette lettre avec les grands textes philosophiques de Jacques Derrida et de Gilles Deleuze, répond l'éditeur à ces nombreuses questions dans sa présentation de Cixoux sous X. D'un coup le nom. Les directrices de ce collectif, Marie-Dominique Garnier et Joana Masó, proposent de revisiter l'œuvre d'Hélène Cixous depuis ses commencements jusqu'aux publications les plus récentes: de Partie (1976) au Voisin de zéro: Sam Beckett et Si près (2007), en passant par Un vrai jardin (1971), Manhattan (2002) ou L'Amour même dans la boîte aux lettres (2005). Le départ de ces analyses est une journée d'études qui s'est tenue à l'Université Paris 8 en 2007 et lors de laquelle plusieurs lectures publiques ont été, on le devine aisément, élaborées autour du nom, celui de l'auteure au X central, en lien avec d'autres : Poe, saint Augustin, Beckett, Kafka, Stendhal, etc. Le livre est séparé en quatre parties, dont la première, «Lectures au près du nom », présente deux textes, le premier rédigé par Marie-Dominique Garnier et le second par Joana Masó. Jean-Michel Rabaté et Marta Segarra signent les deux textes de la seconde partie, intitulée «Boîtes aux lettres diacritiques », alors que Frédéric Regard et Isabelle Hersant étudient chacun les correspondances de l'écrivain avec des artistes contemporains : Simon Hantaï, Roni Horn et Maria Chevska dans la troisième section («De H. C. à Hantaï et Chevska »). Enfin, Laurent Milesi se consacre à l'étude du Voisin de zéro alors que Nadia Setti s'intéresse à ce qu'elle nomme les «mélographies» de Cixous dans le quatrième et dernier chapitre, «Voiser, voisiner : traductions, transports ».

Toutes les lectures qui figurent dans Cixous sous $X$ tournent autour du nom, posent la question de la nomination, articulent le problème de la signature ou celui du centre. En 
effet, qu'il y a-t-il au milieu du nom ? Quel est ce X qui trône au centre du nom? «Est-ce un territoire? Une déterritorialisation ? Ce X est-il une fin d'alphabet? Un souvenir hiéroglyphique ? Une invitation à la réversibilité, du ver au rêve, de la rune à l'urne? Sortie ou commencement? [...] Une synthèse, en plein milieu d'un nom ? Une sainte thèse en forme de croix ?» (19), s'interroge Marie-Dominique Garnier dans un texte intitulé « Lettres voilées : de Partie (Hélène Cixous) à Sauf le nom (Jacques Derrida) ». Dans Cixous sous X. D'un coup le nom, tous les auteurs regardent sous les mots pour voir ce qui s'y trouve; ils vont derrière le $\mathrm{X}$, jouent avec les contiguïtés sémantiques et lexicales. C'est ce que fait Marie-Dominique Garnier, qui au demeurant s'amuse peut-être plus que les autres contributeurs du recueil avec les mots, leur étymologie, avec le polylinguisme: "mues/murs» (31); «tombe/ texte » (31); «boîte à lettres/boîtes à couture » (31) ; « œil/écueil » (21) ; «terre/taire » (22) ; « Oran/hors-en » (19) ; «il ou aile» (19), etc. Comme le savent les lecteurs de Partie, il s'agit d'un procédé d'écriture propre à Cixous. En effet, les mots d'Hélène Cixous s'infiltrent constamment sous d'autres mots, entre les lexèmes; ils trouvent des passages entre les sens et parviennent à se multiplier, et c'est ce que fait Garnier dans ce texte dialogique entre Partie et Saufle nom.

Joana Masó, traductrice en espagnol des ouvrages de Cixous et de Derrida, signe le second texte, intitulé "Entre citation et devenir : noms verbaux d'Hélène Cixous », à partir d'une citation de Clarice Lispector — « Ce que je dis n'est jamais ce que je dis mais autre chose $»-$, citation qui nous permet d'entrer dans le vif du sujet : le jardin cixousien, c'est-à-dire le Vrai jardin. Il y est question de faire «résonner la lecture derridienne de la théologie négative » (40), d'offrir au lecteur 
une approche négative du jardin biblique. Par conséquent, cela inclut que le lecteur connaît bien ce concept de théologie négative de Derrida.

Avec « Le tréma de Poe, l'Umlaut d'Ulm et le circonflexe de Futur », Jean-Michel Rabaté produit sans doute le texte le plus riche du collectif. Pour lui, qui rend un hommage à Cixous - «Il m'a donc semblé que pour lui rendre hommage il fallait que je me fasse encore plus littéral, que je m'attache à la ponctuation de son écriture» (56) - , chaque lecteur de l'écrivaine participe au texte en ponctuant sa lecture puisqu'il doit sans cesse « se livrer à une interprétation, faute de quoi des mots le submergent » (57).

"Les lieux sont fondamentaux chez Cixous », c'est ainsi que débute l'article de Marta Segarra («Les lettres de l'amour... même »), laquelle s'intéresse à L'Amour même dans la boîte aux lettres, un texte de Cixous qui s'attache beaucoup à la pensée de Derrida: " il y a aussi dans L'Amour même du "secret" et du "problème" - dans le sens derridien des deux termes ", affirme Segarra. Suis-je une mauvaise lectrice? Je n'ai jamais compris quel était le sens derridien du secret et du problème dans cette analyse de L'Amour même.

Frédéric Regard, pour sa part, sait voir et montrer en quoi consiste la richesse de l'écriture de Cixous, en quoi, chez elle, l'essai et la théorie toujours se transforment en confessions. L'idée d'une faute originelle semble hanter l'œuvre de Cixous, c'est du moins ce que démontre Regard dans un très beau texte, intitulé "Un effet de Manche : lecture de "K. A Notebook" de Maria Chevska et Hélène Cixous ». Ainsi, Regard formule cette judicieuse remarque sur le procédé d'écriture de celle-ci : «Si les textes de Cixous s'essaient toujours à une vérité nouvelle, 
[...] c'est aussi que les actants sont soumis à d'incessantes métamorphoses, transformant la scène d'interlocution en une scène polyphonique, dont le sens premier est toujours parasité par l'instabilité des instances, imprévisibles et poreuses » (119). Regard joue ici avec le fait que $K$ est une œuvre qui tourne autour d'une lettre qui rappelle au lecteur le X de Cixous et qui le mène au $\mathrm{K}$ de Kafka.

Les lecteurs du Tablier de Simon Hantaï. Annagrammes de Cixous et de L'Étoilement. Conversation avec Hantaï, de Georges Didi-Huberman se retrouveront aisément dans le texte d'Isabelle Hersant, "Sous la figuration du trait, la figure du retrait : le pli et le secret». Cette étude s'attache à la «trinité » (132) «table, tablier, tableau » (132) qui « porterait la marque quasi œdipienne - le tablier venant comme l'enfant de la scène primitive, encadré qu'il se trouve entre la table et le tableau » (132). Il y a effectivement, dans Le Tablier de Simon Hantaï, ce " glissement d'un terme à l'autre » (132) « de la table au tablier, et du plan au pan » (132) dans lequel «s'énonce la figure du pli ». En somme, Cixous élabore, quelques années après DidiHuberman, le travail sur l'empreinte de l'objet primordial, et c'est ce que fait très bien ressortir Hersant.

Dans la quatrième et dernière section, Laurent Milesi prend comme point de départ de sa réflexion cette question fondamentale que pose l'un des deux frères jumeaux de Finnegans Wake: «When is a man not a man ? » Quand c'est un imposteur, répond l'autre frère dans un jeu de mots difficile à rendre dans la traduction française. Quand il est «plus » qu'un homme, moins qu'un homme, quand c'est un «simple matricule», «un moins que rien», un «simple résidu numérique », «à peine plus qu'un nul (0)» (149). Ou encore, 
comme dans la "mathématique existentielle beckettocixousienne, un moins que néant», affirme Milesi, citant Le Voisin de zéro. Sam Beckett de Cixous. «Algébrie à l'irlandaise : Le Voisin de zéro », comme on le devine, s'intéresse à l'algèbre (l'algèbre négative, entre autres), et conclut qu' « être voisin de zéro, c'est non seulement être la virgule, typographiquement proche du point, qui en est sa traduction en anglais dans les nombreux nombres décimaux, par exemple dans 0,999» (158), c'est aussi être cet intraduisible de l'écriture cixousienne.

Signé Nadia Setti, le dernier texte du recueil, «Mélographies cixousiennes : partitions pour lettres enfouies", revient une dernière fois sur le $\mathrm{X}$ du nom. Dans l'œuvre de Cixous, rappelle Setti, les mots (comme ceux qui forment le nom/non) ne sont pas stables et invariants : «le nom n'est pas figé par la Loi, ni cette entité généalogique et paternelle qui menace sans cesse la possibilité d'être autre, mais est au contraire décomposable et dispersable à l'infini, source d'innombrables réécritures, défiant les lois et les limites du propre et du commun » (173). En mai 2003, lors du colloque intitulé Genèses Généalogies Genres, qui vit la remise par Hélène Cixous de l'ensemble de ses manuscrits à la Bibliothèque nationale de France, la principale intéressée se disait porteuse d'un ensemble de commencements et de fins : "je suis une descendante de morts. [...] Mes morts vivent dedans moi», écrivait-elle. Comme l'affirme Setti, « avant que les lettres du nom apparaissent il aura fallu toute une trajectoires de la "trace sonore" mais, une fois apparu, le nom n'efface pas la voix » (175). Si les morts de Cixous vivent en elle, sous elle ou en son centre, ils lui ont aussi transmis le $\mathrm{X}$ du nom, le nom qui raconte 
une trajectoire, la sienne, mais pas uniquement: cette trace sonore est la langue de la femme sauvage et de ses rêveries.

"Intouchable. Un nom dit toujours non, qu'on le veuille ou non. » C'est ainsi que débute l'incipit de Cixous sous $X$. D'un coup le nom, un ouvrage pour les initiés et les connaisseurs de Cixous, de son langage, de ses œuvres, de ses amitiés et de ses influences. On remarque souvent que les spécialistes de l'œuvre de Cixous, qu'ils soient, comme c'est le cas ici, des universitaires, ou des critiques, semblent toujours être d'abord et avant tout et, peut-être, davantage des admirateurs de Cixous que des critiques de l'œuvre. Une des conséquences en est que les contributeurs adoptent le style de l'auteure et acceptent d'emblée toutes ses opinions, toutes ses théories ou tous ses concepts. Malheureusement, l'admiration manifeste, globale et inconditionnelle pour un auteur peut générer des questions sur l'esprit critique de ceux qui en analysent l'œuvre. 